\title{
Does atraumatic restorative treatment reduce dental anxiety in children? A systematic review and meta-analysis
}

\author{
Arun K. Simon ${ }^{1,2}$, T. V. Bhumika ${ }^{2}$, N. Sreekumaran Nair ${ }^{3}$
}

Correspondence: Dr. N. Sreekumaran Nair

Email: sree.nair@manipal.edu

\begin{abstract}
'Department of Public Health Dentistry, Manipal College of Dental Sciences, Mangalore, Manipal University, Karnataka, India,

2Department of Statistics, Public Health Evidence South Asia, Manipal University, Manipal, Karnataka, India,

${ }^{3}$ Department of Statistics, Dr. TMA Pai Endowment Chair in Systematic Reviews and Evidence Based Public Health, Manipal University, Manipal, Karnataka, India
\end{abstract}

\section{ABSTRACT}

Dental anxiety is one of the major problems affecting children, which impairs the rendering of dental care, leading to impaired quality of life. It often leads to occupational stress in dental personnel and conflict between parents/caregivers. The objective of this study was to conduct a systematic review and meta-analysis of randomized controlled trials done in children, to synthesize evidence of the effectiveness of atraumatic restorative treatment (ART) in reducing dental anxiety in children compared to conventional restorative treatments. The databases searched included PubMed, Google Scholar and The Cochrane Oral Health Group's Trials Register. Eligible studies reporting dental anxiety by a variety of psychometric scales were tabulated. The review was conducted and reported in accordance with the guidelines provided by the Cochrane Collaboration. Among 416 studies retrieved through literature search, six studies matched the inclusion criteria. Due to lack of data, only three studies were included for meta-analysis using RevMan software (Review Manager, Version 5.3; The Cochrane Collaboration, Copenhagen, 2014). The pooled meta-analysis data, (standardized mean difference -2.12 [95\% confidence interval: $-4.52,0.27]$ ) failed to show any difference between ART group and the conventional treatment group. In conclusion, ART was not more beneficial in reducing dental anxiety among pediatric dental patients. The findings are relevant in the field of clinical practice in dentistry in the management of the anxious pediatric dental patient.

Key words: Child, dental restoration, oral health, panic, practice guideline, test-anxiety scale

\section{INTRODUCTION}

Parents and dentists assume a crucial part in making the dental visit a pleasant experience for the child. There is no greater impediment to provide oral health care than an anxious pediatric patient in the dental office. Dental anxiety is "a feeling of apprehension about dental treatment that is not necessarily connected to a specific external stimulus." [1] The terms, "dental anxiety," "dental fear" and "dental phobia" have been used interchangeably in the literature with no clear demarcation between them. ${ }^{[2,3]}$ Dental anxiety can have its origin in childhood, ${ }^{[4]}$ and it can obstruct the professional work of the dental personnel. ${ }^{[5]}$ Dental anxiety in children is also associated with lower utilization ${ }^{[6]}$ and poorer oral health. ${ }^{[7-9]}$ Anguish during dental appointments are common among patients with dental anxiety, and they are less likely to comply with instructions from the dentist. ${ }^{[10,11]}$ Irrespective of the cultural background or the country, dental anxiety can range between

How to cite this article: Simon AK, Bhumika TV, Nair NS. Does atraumatic restorative treatment reduce dental anxiety in children? A systematic

review and meta-analysis. Eur J Dent 2015;9:304-9.

Copyright $\odot 2015$ Dental Investigations Society.

DOI: $10.4103 / 1305-7456.156841$ 
$4 \%$ and $20 \%$ in the population. ${ }^{[2]}$ Patients with dental anxiety are also more likely to be referred for sedation to carry out dental treatment. ${ }^{[12,13]}$ Development of nonpharmacological interventions to reduce dental anxiety has important dental public health significance in preventing over utilization of emergency dental services. ${ }^{[14]}$

The conventional or traditional restorative approaches involve the use of rotary handpieces, dental burs and local anesthesia for pain relief. The restorative materials used can vary from dental amalgam to various resin-based restorations. The discomfort associated with conventional cavity preparation makes patients avoid seeking dental care. ${ }^{[15]}$ In addition to this, the sensitivity associated with cutting of dentin, high pitched noises and vibration leads to the apprehension in the patients. ${ }^{[16]}$ The four major sensory triggers for dental anxiety in the dental office are smells (e.g. eugenol and cut dentine), sights (e.g. needles, air turbine drills), sounds (drilling) and sensations (high frequency vibrations). The " $4 S^{\prime}$ " principle involves removing these triggers to reduce dental anxiety among patients in the dental office. ${ }^{[17]}$

The atraumatic restorative treatment (ART) was pioneered in the mid 1980's in Tanzania. It encompasses the concept of minimal intervention approach for treating carious teeth. The ART approach is a "procedure based on removing carious tooth tissues using hand instruments alone and restoring the cavity with an adhesive restorative material".${ }^{[18]}$ The properties of fluoride release and pulpal biocompatibility make the glass ionomer cement, the material of choice in the ART approach. ${ }^{[19]}$ It is particularly helpful in the treatment of an apprehensive pediatric patient since it does not involve the use of local anesthesia and rotary handpieces. ${ }^{[20]}$ The resultant smaller cavities in the ART approach helps to conserve tooth structure, ${ }^{[21]}$ and it is useful to minimize discomfort among both children and adults. ${ }^{[22]}$ ART may reduce dental anxiety by removing the sensory triggers.

However, to the best of our knowledge, we have not come across any systematic review providing evidence on the effectiveness of ART compared to conventional restorative treatment on dental anxiety in children. Hence, we conducted this systematic review under the methodology of Cochrane reviews to assess the effectiveness of ART compared with conventional restorative treatment in primary teeth on dental anxiety of children.

\section{MATERIALS AND METHODS}

The systematic review was carried out using the Cochrane Collaboration methodology described in the Cochrane Handbook for systematic reviews of interventions. ${ }^{[23]}$ The study protocol was prepared following the Cochrane structure, and then the systematic review was carried out according to the protocol. Both randomized controlled trials (RCTs) and controlled clinical trials (CCTs) of studies comparing the effectiveness of ART against conventional restorative treatment on reducing dental anxiety using any psychometric scales were included. We restricted our search to English language journals only. Case reports or case series, observational studies, review articles/letters and studies in which dental anxiety was not measured were excluded from the review.

\section{Types of participants}

Studies that included both sexes, aged within 15 years, with at least one carious primary tooth, who have undergone restoration with either ART or Conventional treatment approaches were included in this review. Patients under long-term medication for systemic illness and syndromic patients were excluded.

\section{Types of interventions}

Interventions are primary teeth that were restored using ART approach with the use of only hand instruments and adhesive material. Modifications of ART approaches that are considered modifications of the original technique proposed were not considered.

\section{Control}

Controls were comparable patients, who received a variety of restorations using conventional cavity preparations using rotary instruments, dental burs and administration of local anesthesia when needed.

\section{Outcome measures}

Dental anxiety of the patient measured by psychometric scales after dental treatment was the primary outcome. Patients discomfort during the procedure and adverse events were considered as secondary outcomes.

\section{Information sources}

Attempts were made to identify relevant published studies in English language journals. The bibliographic databases included PubMed, Google Scholar and The Cochrane Oral Health Group's Trial Register. These databases were searched for the period from 1980 to end of August 2014 to identify studies to be considered for this review. Reference lists of identified studies and relevant reviews were further examined to identify 
studies not captured during the initial literature search. We did not undertake hand searching of journals. Clarifications were obtained from corresponding authors in case of unclear methods or missing data.

\section{Search strategy and data extraction}

Two review authors (A.K.S and T.V.B) carried out the literature search independently. The search strategy included appropriate keywords combined with Boolean operators "AND" and "OR". The search string for PubMed were as follows, ([[[[[][[Randomized Controlled Trial] OR RCT] OR Quasi trials] OR CCT] OR Controlled Clinical Trial]] AND [[[[[anxiety] OR fear] OR apprehension] OR unease] OR worry]] AND [[[[[ART] OR IRT] OR Atraumatic restorative technique] OR Interim restorative technique] OR ART]] AND [[[[children] OR child] OR young people] OR preschool] OR school going). Disagreements were resolved through consultation with the third review author (N.S.N). The titles of the retrieved articles were screened first followed by the abstracts and then full text. Disagreements at each stage were resolved through consultation with the third review author (N.S.N). One author (A.K.S), prepared data extraction sheet and was checked for correctness by the other authors (T.V.B and N.S.N). The data extraction sheet included the general study information, study setting, the interventions, inclusion and exclusion criteria and outcome characteristics. Two authors (A.K.S and T.V.B) did the data extraction independently.

\section{Risk of bias of individual studies}

The Cochrane risk of bias tool was used for the assessment of risk of bias of eligible studies. ${ }^{[24]}$ It was carried out by two authors independently (A.K.S and T.V.B). Discussions among all the authors were used to reach consensus in case of disagreements.

\section{Data synthesis}

The standardized mean difference (SMD) was used as a summary statistic in meta-analysis since different studies measured the outcome by use of different psychometric scales. The $I^{2}$ index was used to assess the statistical heterogeneity, with the level of significance at $P<0.01$. We used a random effect meta-analysis to address heterogeneity. Meta-analyses were undertaken in Review Manager. ${ }^{[25]}$ Results were shown as forest plot of SMD and its 95\% confidence interval (CI).

\section{RESULTS}

\section{Study selection}

A total of 416 studies were retrieved after literature search [Figure 1] and 405 articles remained after duplicate removal. After the review of titles and abstracts, 10 studies remained for full text reading and data extraction. One study was excluded, since there was no comparison group, two studies were not relevant and one study did not meet the language criteria. The remaining six studies were subjected to complete analysis. ${ }^{[22,26-30]}$ A summary of the characteristics of the included study is provided in Table 1. Following data extraction, the nonavailability of the values of mean and standard deviation for the psychometric scale values restricted the inclusion of three studies in the meta-analysis. ${ }^{[26,29,30]}$ The author follow-up was unsuccessful, and they can be included in the meta-analysis at a further date.

\section{Study description}

Diverse studies were included in this systematic review [Table 1], with two studies reported from Brazil and one each from Indonesia, Turkey, India

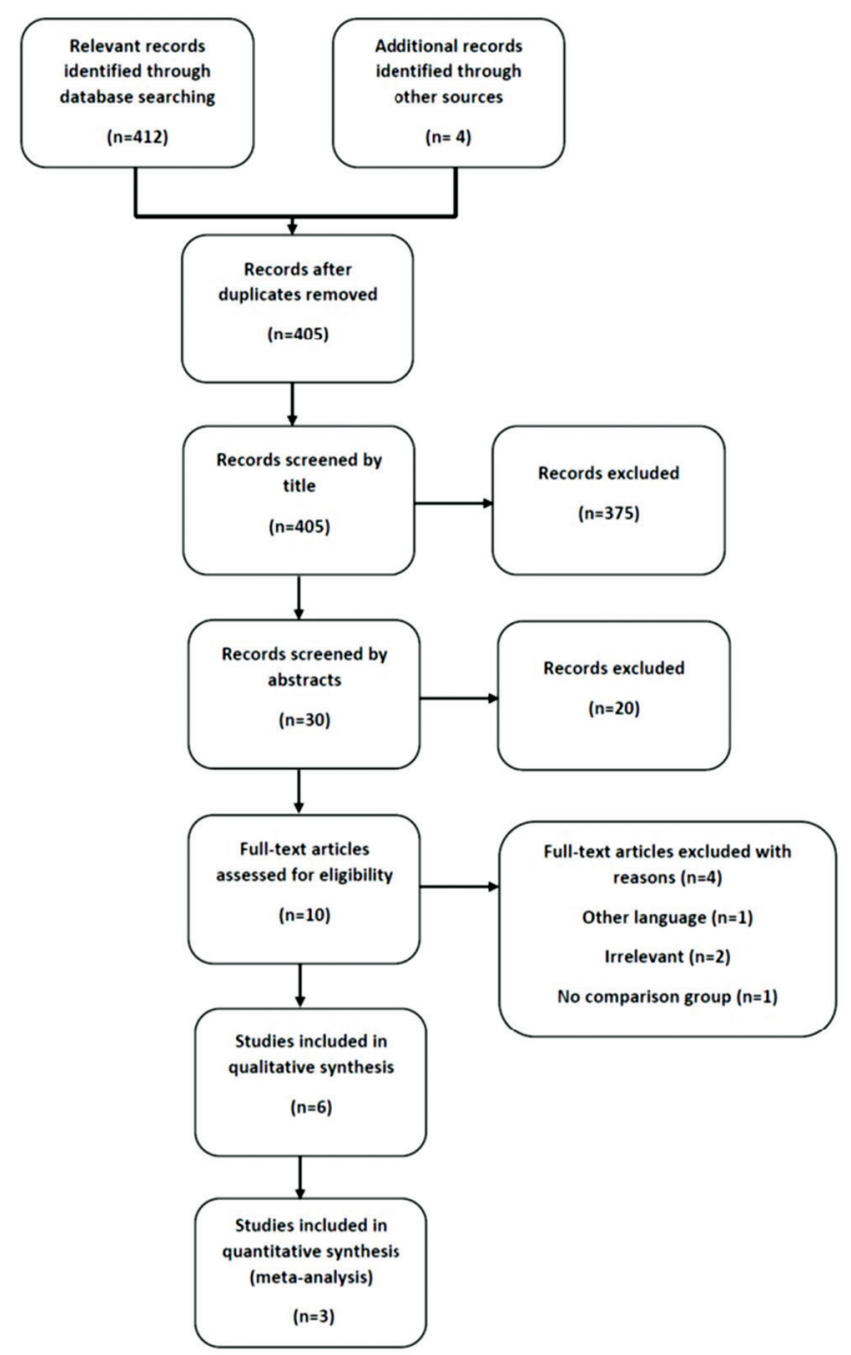

Figure 1: Flow-chart of the selection of studies for the systematic review of the effectiveness of atraumatic restorative treatment approach in reducing dental anxiety in children (PRISMA chart) 


\begin{tabular}{|c|c|c|c|c|c|c|}
\hline Study & Design & Country & Setting & Participants & Age & Scales used \\
\hline Schriks and van Amerongen & RCT & Indonesia & Not clear & 403 & 6 years & Modified Venham scores \\
\hline De Menezes Abreu et al. & ССТ & Brazil & School & 211 & 6-7 years & FIS \\
\hline Topaloglu-AK et al. & $\mathrm{RCT}$ & Turkey & Hospital & 160 & $6-7$ years & VPT \\
\hline Luz et al. & $\mathrm{RCT}$ & Brazil & Hospital & 30 & $4-7$ years & FIS \\
\hline Goud et al. & $\mathrm{RCT}$ & India & Hospital & 200 & $6-8$ year & Modified Venham scores \\
\hline Mickenautsch et al. & $\mathrm{RCT}$ & South Africa & Hospital & 143 & Mean age between 6-7 years & CFSS-SF \\
\hline
\end{tabular}

and South Africa. The studies were carried out in either hospital or school settings. Five articles did not mention sources of funding and one study was funded by the University. The studies included were full text articles published in English language from 2003 to 2012. Five of the included six studies were RCTs and one study was CCT. All the studies measured dental anxiety, using psychometric scales. The psychometric scales used were the Modified Venham Scores, Facial Image Scale, the Venham Picture Test and the Short Form of the Dental Subscale of the Children's Fear Survey Schedule. Other outcome measures reported in studies included dental pain and length of time taken for treatment. No studies reported the occurrence of adverse events.

\section{Risk of bias assessment}

Risk of bias was estimated for all the included studies [Figure 2]. Although there was a report of random allotment of the study interventions, only one study out of the six had specified the procedurefollowed. Measures to ensure blinding of participant and assessors and the procedures for allocation concealment were not adequately addressed in most of the studies.

\section{Synthesis of results}

Based on 514 participants in the intervention and control groupsfrom3studies, thesummarySMDforARTapproach reducing dental anxiety was -2.12 (95\% CI: $-4.52,0.27)$, with high statistical heterogeneity (99\%) [Figure 3]. Since the pooled meta-analysis results showed significant heterogeneity $\left(P<0.00001, I^{2}=99 \%\right)$, random effect model was used.

\section{DISCUSSION}

With this systematic review, we aimed to evaluate, the effectiveness of ART compared with conventional restorative treatment in primary teeth on dental anxiety of children. The main finding of this study was that both the treatment approaches were comparable in reducing dental anxiety among children. This is one

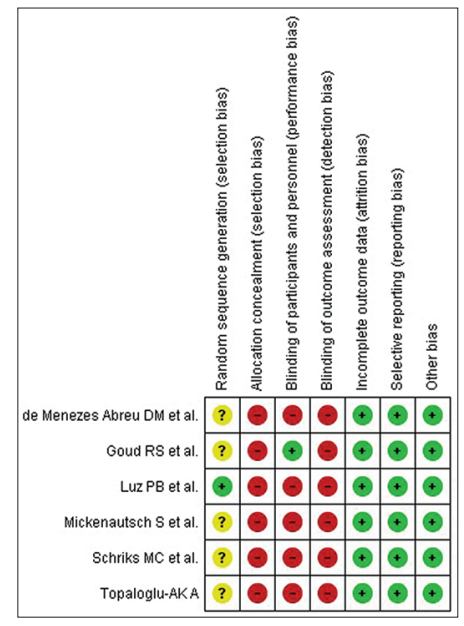

Figure 2: Assessment of risk of bias of studies for the systematic review of the effectiveness of atraumatic restorative treatment approach in reducing dental anxiety in children

of the first studies, which provides evidence for this aspect of ART. As many as six publications were found after the literature search, which included acceptable homogenous patient groups. Dental anxiety has shown to increase the pain perception of the pediatric patient, irrespective of the anesthetic devices used. ${ }^{[31]}$ Conversely, uncomfortable dental treatments can also result in increased dental anxiety. ${ }^{[32]}$ Pain is reported commonly during the restorative procedure, especially when no local analgesia has been used prior to treatment. The ART approach provides an alternative approach to minimal trauma, conservation of tooth structure and avoidance of local anesthesia, which has been welcomed by both pediatric and adult patients. ${ }^{[33]}$ Studies have shown that the patient becomes more receptive toward treatment with the ART approach in comparison with the conventional treatments. ${ }^{[34]}$ Some of the possible reason for anxiety in the dental settings may be, the depth of the cavity, instruments in the operatory, sounds and vibrations, psychology of the child and previous dental treatment experiences. ${ }^{[35]}$ The use of the ART approach by a trained professional may eliminate these problems and can be a positive influence toward future dental 
Simon, et al: : Atraumatic restorative treatment and dental anxiety

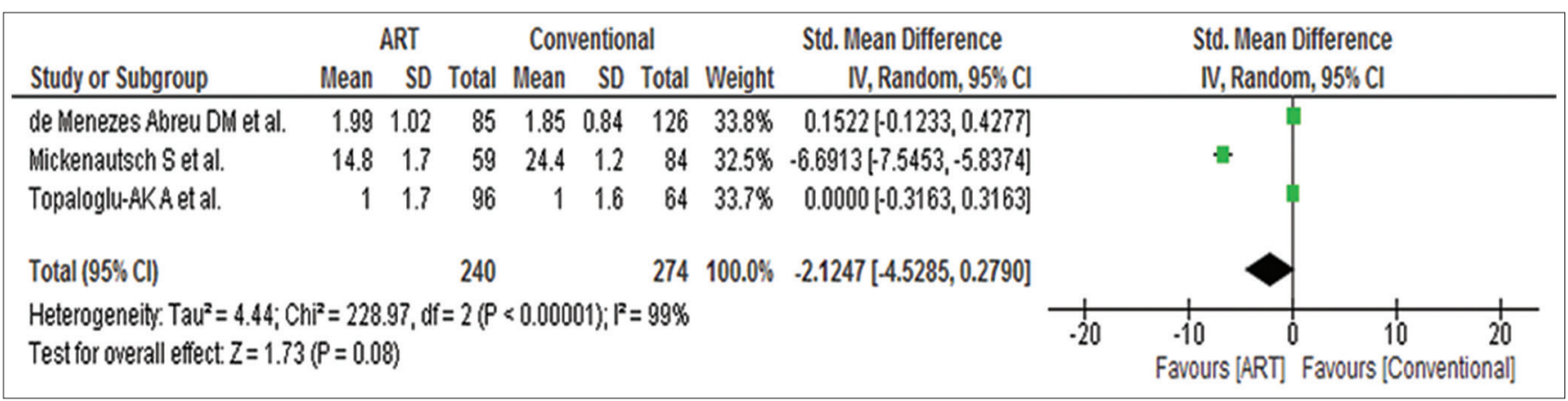

Figure 3: Forest plot of standardized mean differences between atraumatic restorative treatment approach and conventional treatment approach in reducing dental anxiety in children

treatments. It is important to reduce anxiety not only in the highly anxious patient who avoids dental care but also in patients who undergo regular dental care in spite of being anxious. The target of any dental treatment should be to prevent the patient adopting a negative dental care behavior. ${ }^{[36]}$

Since, standardized measures were lacking to measure dental anxiety, the meta-analysis was completed using the SMD across studies evaluating the same interventions. The evidence from the pooled meta-analysis suggests that the ART approach and the conventional restorative approach were comparable in reducing dental anxiety among children. The result of the present study can be explained by the fact that the correct way of using psychometric scales is patient dependent. ${ }^{[37]}$ In addition, heterogeneity may arise due to the control groups subject to different treatments. The results also can be interpreted through the many limitations present in this study. One of the primary limitations of the present study was the inadequate number of studies that matched our inclusion criteria. There is paucity of well-designed intervention studies in this area of ART. Another limitation was that of the lack of information pertaining to the related study variables, which prevented us from analyzing all of the included studies. The poor reporting of results and lack of clarity of the extent of randomization, allocation concealment and blinding done also had an impact on the study results. Standardized reporting guidelines for intervention studies can reduce the significant knowledge gaps identified. The language used and the limited number of databases searched can be considered as a drawback of this study.

\section{CONCLUSION}

Even though, the ART approach is patient friendly, the analysis of retrieved studies showed that there is no difference between the ART approach and the conventional approach in reducing dental anxiety in children. The greatest impact of this study is in the area of clinical practice, for the management of pediatric dental patients. In spite of the few studies included, we can conclude that ART was not more beneficial in reducing dental anxiety among pediatric dental patients. On the other hand, there are several aspects related to dental anxiety that can be investigated in detail. More number of high-quality RCTs are needed to conclude the efficacy of reducing dental anxiety using the ART approach.

\section{ACKNOWLEDGMENT}

We would like to acknowledge the help provided by Mr. Ravi Shankar, Research Scholar, Department of Statistics, Manipal University for carrying out this study.

\section{REFERENCES}

1. Folayan MO, Idehen EE, Ojo OO. The modulating effect of culture on the expression of dental anxiety in children: A literature review. Int J Paediatr Dent 2004;14:241-5.

2. Skaret E, Soevdsnes EK. Behavioural science in dentistry. The role of the dental hygienist in prevention and treatment of the fearful dental patient. Int J Dent Hyg 2005;3:2-6.

3. Coric A, Banozic A, Klaric M, Vukojevic K, Puljak L. Dental fear and anxiety in older children: An association with parental dental anxiety and effective pain coping strategies. J Pain Res 2014;7:515-21.

4. Locker D, Liddell A, Dempster L, Shapiro D. Age of onset of dental anxiety. J Dent Res 1999;78:790-6.

5. Pine CM, Adair PM, Burnside G, Nicoll AD, Gillett A, Borges-Yáñez SA, et al. Barriers to the treatment of childhood caries perceived by dentists working in different countries. Community Dent Health 2004;21:112-20.

6. Milgrom P, Mancl L, King B, Weinstein P, Wells N, Jeffcott E. An explanatory model of the dental care utilization of low-income children. Med Care 1998;36:554-66.

7. Armfield JM, Slade GD, Spencer AJ. Dental fear and adult oral health in Australia. Community Dent Oral Epidemiol 2009;37:220-30.

8. Nicolas E, Bessadet M, Collado V, Carrasco P, Rogerleroi V, Hennequin M. Factors affecting dental fear in French children aged 5-12 years. Int J Paediatr Dent 2010;20:366-73.

9. Klingberg G, Berggren U, Carlsson SG, Noren JG. Child dental fear: Cause-related factors and clinical effects. Eur J Oral Sci 1995;103:405-12.

10. Van Houtem CM, Aartman IH, Boomsma DI, Ligthart L, Visscher CM, de Jongh A. Is dental phobia a blood-injection-injury phobia? Depress Anxiety 2014;31:1026-34.

11. Beena JP. Dental subscale of children's fear survey schedule and dental 
caries prevalence. Eur J Dent 2013;7:181-5.

12. Boyle CA, Newton T, Milgrom P. Who is referred for sedation for dentistry and why? Br Dent J 2009;206:E12.

13. Mahesh R, Prasad V, Menon PA. A case of accidental aspiration of an endodontic instrument by a child treated under conscious sedation. Eur J Dent 2013;7:225-8

14. Kanegane K, Penha SS, Borsatti MA, Rocha RG. Dental anxiety in an emergency dental service. Rev Saude Publica 2003;37:786-92.

15. Berggren U, Meynert G. Dental fear and avoidance: Causes, symptoms, and consequences. J Am Dent Assoc 1984;109:247-51.

16. Banerjee A, Watson TF, Kidd EA. Dentine caries excavation: A review of current clinical techniques. Br Dent J 2000;188:476-82.

17. Walsh LJ. Anxiety prevention: Implementing the $4 \mathrm{~S}$ principle in conservative dentistry. Auxilliary 2007;17:24-6.

18. Frencken J, van Amerogen E, Phantumvanit P, Songpaisan $Y$, Pilot T. Manual for the Atraumatic Restaurative Treatment Approach to Control Dental Caries. $3^{\text {rd }}$ ed. Groningen: WHO Collaborating Centre for Oral Health Research; 1997.

19. Smales RJ, Yip HK. The atraumatic restorative treatment (ART) approach for primary teeth: Review of literature. Pediatr Dent 2000;22:294-8.

20. Smales RJ, Yip HK. The atraumatic restorative treatment (ART) approach for the management of dental caries. Quintessence Int 2002;33:427-32.

21. Togoo RA, Blower C. Atraumatic Restorative Treatment (ART) in pediatric dentistry examination gloves. Int J Clin Dent Sci 2011;2:30-5.

22. Mickenautsch S, Frencken JE, van't HM. Atraumatic restorative treatment and dental anxiety in outpatients attending public oral health clinics in South Africa. J Public Health Dent 2007;67:179-84.

23. Higgins JP, Green S, editors. Cochrane Handbook for Systematic Reviews of Interventions. Version 5. The Cochrane Collaboration; 2011. Available from: http://www.handbook.cochrane.org/. [Last accessed on 2014 Sep 05].

24. Higgins JP, Altman DG, Gøtzsche PC, Jüni P, Moher D, Oxman AD, et al. The Cochrane Collaboration's tool for assessing risk of bias in randomised trials. BMJ 2011;343:d5928.

25. Review Manager (RevMan) [Computer program]. Version 5.3. Copenhagen: The Nordic Cochrane Centre, The Cochrane Collaboration, 2014.

26. Schriks MC, van Amerongen WE. Atraumatic perspectives of ART: Psychological and physiological aspects of treatment with and without rotary instruments. Community Dent Oral Epidemiol 2003;31:15-20.

27. De Menezes Abreu DM, Leal SC, Mulder J, Frencken JE. Dental anxiety in 6-7-year-old children treated in accordance with conventional restorative treatment, ART and ultra-conservative treatment protocols. Acta Odontol Scand 2011;69:410-6.
28. Topaloglu-Ak A, Eden E, Frencken JE. Perceived dental anxiety among schoolchildren treated through three caries removal approaches. J Appl Oral Sci 2007;15:235-40.

29. Luz PB, Barata JS, Meller CR, de Slavutsky SM, de Araujo FB. ART acceptability in children: A randomized clinical trial. Rev Fac Odontol Porto Alegre 2012;53:27-31.

30. Goud RS, Nagesh L, Shoba F, Raju HG. Assessment of discomfort experienced by school children while performing 'ART' and ' $\mathrm{MCP}^{\prime}$-An experimental study. J Dent (Tehran) 2012;9:229-37.

31. Kuscu OO, Akyuz S. Is it the injection device or the anxiety experienced that causes pain during dental local anaesthesia? Int J Paediatr Dent 2008;18:139-45.

32. De Menezes Abreu DM, Leal SC, Mulder J, Frencken JE. Pain experience after conventional, atraumatic, and ultraconservative restorative treatments in 6-to 7-yr-old children. Eur J Oral Sci 2011;119:163-8.

33. Rahimtoola S, van Amerongen E, Maher R, Groen H. Pain related to different ways of minimal intervention in the treatment of small caries lesions. ASDC J Dent Child 2000;67:123-7, 83.

34. Frencken JE, Holmgren CJ. ART: A minimal intervention approach to manage dental caries. Dent Update 2004;31:295-8, 301.

35. Carvalho TS, Ribeiro TR, Bönecker M, Pinheiro EC, Colares V. The atraumatic restorative treatment approach: An "atraumatic" alternative. Med Oral Patol Oral Cir Bucal 2009;14:e668-73.

36. Wide Boman U, Carlsson V, Westin M, Hakeberg M. Psychological treatment of dental anxiety among adults: A systematic review. Eur J Oral Sci 2013;121:225-34.

37. Dixon KE, Thorn BE, Ward LC. An evaluation of sex differences in psychological and physiological responses to experimentally-induced pain: A path analytic description. Pain 2004;112:188-96.

\begin{tabular}{|l|l|}
\hline \multicolumn{3}{|c|}{ Access this article online } \\
\hline Quick Response Code: & $\begin{array}{l}\text { Website: } \\
\text { www.eurjdent.com }\end{array}$ \\
\cline { 2 - 3 } & $\begin{array}{l}\text { Source of Support: We gratefully } \\
\text { acknowledge the support of Manipal } \\
\text { University through Dr.TMA Pai Endowment } \\
\text { Chair on Systematic review and Evidence } \\
\text { based public health and Public Health } \\
\text { Evidence South Asia (South Asian Satellite of } \\
\text { Cochrane Public Health Group) to carry out } \\
\text { this work. Conflict of Interest: None declared. }\end{array}$ \\
\hline
\end{tabular}

